\title{
Generalizing everyday memory: Signs and handedness
}

\author{
MARYANNE MARTIN \\ University of Oxford, Oxford, England \\ and \\ GREGORY V. JONES \\ University of Warwick, Coventry, England
}

\begin{abstract}
Memory for frequently encountered road signs was investigated. In Experiment 1, the average level of recall of road sign features was found to be only 47\%. In Experiment 2, more left-handed than righthanded people recalled that a walking figure faces right on one sign, whereas more right-handed than left-handed people recalled that a digging figure faces left on another sign. Performance thus reflected not a difference in level of mnemonic ability between left-handed and right-handed groups but instead the compatibility between group and task. In Experiment 3, participants were asked to draw any figure walking and any figure digging, with a pattern of results similar to that of Experiment 2 . It is suggested that handedness effects in recall are mediated by motor imagery.
\end{abstract}

The study of memory for experimentally presented multicomponent stimuli has a considerable history (see, e.g., Bower, 1994; Jones, 1976). A much briefer history attaches to the study of memory for multicomponent stimuli encountered in everyday life. Only memory for the appearance of a single type of multicomponent stimulus, the coin, has been explored at all widely (see, e.g., Jones, 1990; Martin \& Jones, 1995; Nickerson \& Adams, 1979; Rubin \& Kontis, 1983). It is thus unclear whether inferences that have been drawn about everyday memory functioning on the basis of such studies possess any validity outside the narrow domain of memory for the appearance of coins. What is needed is to discover whether the most striking aspects of memory for the appearance of coins may also be observed with a completely different type of everyday stimulus.

Two findings concerning memory for the appearance of coins have been particularly striking, in that neither had been predicted on the basis of much more extensive laboratory studies. The first finding is that there is a low level of recall for features that have been viewed repeatedly over a period of years. As many as half of the features may be lost. For example, estimates of recall levels include those of $67 \%$ for eight features of the U.S. penny (Nickerson \& Adams, 1979), $51 \%$ for seven features of the British penny, and $43 \%$ for eight features of the British

The authors are grateful to James C. Bartlett, Morton Ann Gernsbacher, and David Rubin for helpful comments and to Kate Elgar, Angela Emler, Claire Hawkins, Sebastian Marcelin-Rice, Marcus Pearce, and Amy Van Eepoel for help with testing and data coding. Correspondence concerning this article may be addressed to M. Martin, Department of Experimental Psychology, University of Oxford, South Parks Road, Oxford OX1 3UD, England (e-mail: maryanne. martin@psy.ox.ac.uk). 50-pence coin (Jones, 1990). This poverty of retention is surprising when set alongside the well-established observation that people are able to recognize correctly very large numbers of pictures that they have viewed only once in the laboratory (Shepard, 1967; Standing, 1973). The second finding concerning memory for the appearance of coins has been reported only recently. If a coin depicts a person facing to one side, left-handed people and right-handed people differ in their recall of this information. Both McKelvie and Aikins (1993), who studied memory for Canadian coins, and Jones and Martin (1997), who studied memory for British coins, found that left-handed people are significantly more likely than righthanded people to recall that the head faces to the right (which it does on both coinages). Again, this effect of handedness on verbal recall is surprising when set alongside the conclusion from laboratory studies that cognitive processes, with the possible exception of those underlying developmental reading disorder, are generally independent of handedness (see, e.g., Bishop, 1990; Springer \& Deutsch, 1993).

Do these anomalous findings concerning memory for the appearance of coins have general implications for our understanding of human memory, or do they merely constitute isolated anomalies? An influential view (see Banaji \& Crowder, 1989) has been that the results derived from studies of everyday memory suffer from a lack of generality. Thus, an acid test for the importance of both of the highlighted findings is whether they may also be observed outside the context of coins. The present work examines memory for another class of material that people observe repeatedly over many years-road signs.

At first sight, road signs appear to be very different from coins as stimuli for investigating everyday memory. They are much larger, their designs are conveyed by dif- 
ferent colors rather than merely by different contours, and attending to them is vital for personal safety. It is possible that these differences will serve in general to improve memory and in particular to eliminate the striking results found with coins. On the other hand, road signs do have two important factors in common with coins. They have several distinct design components, and some of them depict a person facing to one side. The first of these aspects is exploited in Experiment 1 and the second in Experiment 2.

\section{EXPERIMENT 1}

British road signs are uniform throughout the country and have generally remained unchanged for many yearstheir last major revision was in 1964 (see Department of Transport, 1989). Some signs are, of course, encountered only occasionally. However, in order to establish the upper limits to recall, the present study utilized some of the most important and common signs on British roads.

\section{Method}

Participants. There were 45 participants. They were volunteers who were students at Oxford or Warwick, with average age 20.4 years $(S D=3.0$ years $)$.

Materials. Four of the commonest and most distinctive road signs were selected for the present study. They are shown on the left side of Figure 1 and, from the top of the figure, comprise the signs for Give Way, Roundabout, ${ }^{1}$ Stop, and No Entry. All of the signs have three structural features: a motif (in the center), a shape (overall), and a border (around the edge). Each of these structural features is colored black, white, or red.

Procedure. Each participant was provided with a red and a black pen and was instructed to draw from memory the current British versions of four different traffic signs. They did so in four separate squares, each with sides of $9.5 \mathrm{~cm}$, captioned as follows: Give Way, "The sign at a road junction which means you should halt if necessary"; Roundabout, "The sign which means you are approaching a roundabout"; Stop, "The sign at a road junction which means you should always halt"; and No Entry, "The sign which means you cannot enter a road." Participants were also asked, "Do you have a driving licence?" and "Do you ride a bicycle nowadays?"

\section{Results}

Table 1 shows the frequency of recall for each feature of each stimulus. To obtain a figurative indication of those elements frequently recalled, the method of Rubin and Kontis (1983) was adopted. In their Figure 2, they depicted only those features that were frequently given as responses (see also Figures 1 and 2 of Jones, 1990). Similarly, the right-hand side of Figure 1 here depicts only those features that were recalled by at least $40 \%$ of the participants. It includes several instances of incorrect recall. For the Roundabout sign, $64 \%$ of the participants incorrectly recalled the shape as a circle (instead of a triangle); for the Stop sign, $40 \%$ of the participants incorrectly recalled the motif as colored black (instead of white), $49 \%$ incorrectly recalled the shape as a circle (instead of an octagon), and $58 \%$ incorrectly recalled the shape as white (instead of red).
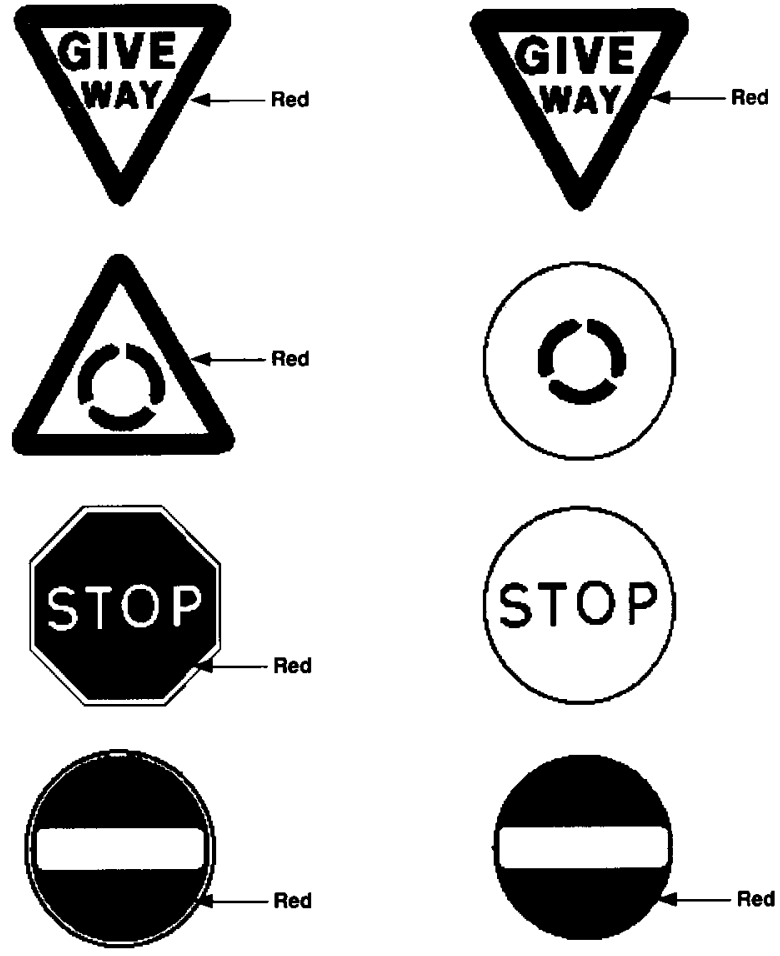

Figure 1. The four signs used in Experiment 1 (left) and the features recalled by $\mathbf{4 0 \%}$ or more of the participants (right).

Summing over the four stimuli and over participants, the mean proportion of structural features (three per stimulus) that were correctly recalled was $48.5 \%$, and the mean proportion of colors (again, three per stimulus) that were correctly recalled was $46.1 \%$. Summing over both structural and color features (six per stimulus), the mean proportion correctly recalled was $47.3 \%$.

The proportions of participants who possessed a driving license and who rode a bicycle were $73 \%$ and $56 \%$, respectively. However, the number of features that a participant recalled did not depend significantly on whether the participant had a driving license $[F(1,43)=1.27]$ or rode a bicycle $[F(1,43)=2.35]$.

\section{Discussion}

The results demonstrated that the level of recall for the appearance of the most common and important road signs is similar to the level of recall for the appearance of coins. The level of recall for six sign features was found here to

Table 1

Recall Levels (\%) for Different Features of Signs

\begin{tabular}{|c|c|c|c|c|c|c|}
\hline \multirow[b]{2}{*}{ Sign } & \multicolumn{2}{|c|}{ Motif } & \multicolumn{2}{|c|}{ Shape } & \multicolumn{2}{|c|}{ Border } \\
\hline & Structure & Color & Structure & Color & Structure & Color \\
\hline Give Way & 67 & 56 & 51 & 82 & 49 & 40 \\
\hline Roundabout & 49 & 82 & 24 & 84 & 31 & 27 \\
\hline Stop & 78 & 20 & 20 & 36 & 31 & 7 \\
\hline No Entry & 67 & 56 & 93 & 60 & 22 & 4 \\
\hline
\end{tabular}




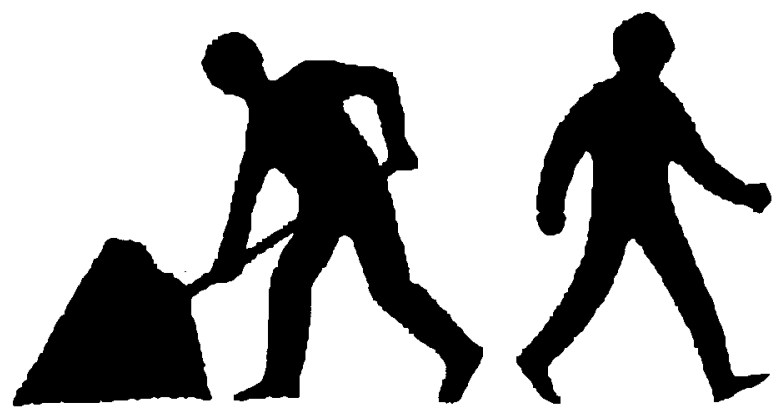

Figure 2. The figures depicted on the Road Works (left) and Pedestrian Crossing (right) signs in Experiment 2.

average only $47 \%$ and to be comparable to levels of recall for seven or eight coin features, which range from $43 \%$ to $67 \%$ (Jones, 1990; Nickerson \& Adams, 1979). Repeated exposure to road signs that have remained constant since 1964 (and, therefore, throughout the entire lives of most of the participants) proved to be remarkably ineffective in ensuring that their appearance can be correctly recalled.

Given the comparability in baseline levels of recall between road signs and coins, it appears appropriate to proceed to investigate the other remarkable finding with coins - that of an effect of handedness on recall. Although evidence exists that handedness is linked with developmental reading disorder (see, e.g., Eglinton \& Annett, 1994) and with the perception of chimeric faces (see, e.g., Luh, Redl, \& Levy, 1994), cognitive performance in general and memory performance in particular have been assumed to be independent of a person's handedness. However, it has recently been demonstrated that this assumption is incorrect. Left-handed people are significantly more accurate than right-handed people at recalling that the heads on British and Canadian coins face to the right (Jones \& Martin, 1997; McKelvie \& Aikins, 1993).

Two different explanations for this finding of an effect of handedness on recall may be distinguished. First, an account in terms of group ability would propose that lefthanded people exhibit a general superiority in recalling information about orientation. Being members of a minority group that comprises only approximately one tenth of the general population, they are likely to have learned to focus more attention on laterality than have the majority right-handed group (e.g., as a consequence of encountering artifacts, ranging from scissors to golf clubs, that are designed for right-handed use). Some support for this possibility is provided by a report by Mebert and Michel (1980) that artists are more likely than the general population to be left-handed. However, it may be noted that this hypothesis of left-handed superiority contrasts with proposals by Levy (1969) of an association between left-handedness and impaired visuospatial performance and by Geschwind and Behan (1982) of an association between left-handedness and a number of prob- lems of ultimately immunological origin (but see Bryden, McManus, \& Bulman-Fleming, 1994).

Second, in contrast, an account of the handedness effect in terms of a compatibility between group and task contends that, depending on the particular task, either left-handed or right-handed people may display superior recall. Within the terms of this compatibility hypothesis, a logical possibility is that, whereas the level of recall of a right-facing stimulus (the heads on British and Canadian coins) was higher for left-handed than for righthanded people, the outcome may be reversed for a leftfacing stimulus. The set of British road signs, unlike the set of British coins, contains members that depict leftfacing as well as right-facing figures. The group ability hypothesis suggests that a single handedness group will recall better both types of figure (on the basis of the coin results, this group will be the left-handed one). In contrast, the compatibility hypothesis suggests that the two types of figure may be recalled better by different groups (on the basis of the coin results, a right-facing stimulus will be better recalled by the left-handed group, and, perhaps, a left-facing stimulus will be better recalled by the right-handed group).

Up to this point, an implicitly dichotomous view of handedness has been assumed. However, handedness may be viewed alternatively as a matter of degree. That is, individuals may be identified not only as left-handed or right-handed but also as members of more specific categories of handedness. There is as yet no general agreement on how these more specific categories should be constituted. However, following M. Annett (1985, 1992), a dichotomous handedness variable will be supplemented in the present work by two graded measures of handedness. Both measures derive from M. Annett's (1985) handedness questionnaire, which elicits hand preference (left, either, or right) for each of 12 different activities. For the first measure, overall handedness is determined by hand preference for writing but is said to be mixed if the other hand is preferred for any of the other 11 activities; if not, the handedness is said to be pure. For the second measure, a handedness index with range 12 (complete right) to 36 (complete left) is obtained by allocating scores of 1,2 , and 3 to right, equal, and left responses, respectively, and then summing over the 12 activities.

\section{EXPERIMENT 2}

\section{Method}

Participants. There were 515 new participants. Categorized on the basis of the hand used for drawing (see Procedure), 198 were left-handed, and 317 were right-handed. They were volunteers who were students or conference attendees at Oxford, Warwick, or Worcester, with average age 26.0 years ( $S D=9.6$ years).

Materials. Road signs are of a uniform design throughout the U.K. (see Department of Transport, 1989). Two common ones have directional human figures as motifs (see Figure 2), as follows: Road Works, the sign to warn of road works ahead shows a figure shoveling to the viewer's left; Pedestrian Crossing, the signal to cross at 
a pedestrian-controlled crossing shows a figure walking to the viewer's right.

Procedure. Each participant was tested using a question booklet with successive items printed on separate pages. For half of the participants, memory was probed first concerning the road works sign and second concerning the pedestrian crossing sign; for the other half, the order was reversed. The probes were as follows: Road Works, "The sign indicating 'road works ahead' has a person digging with a shovel. Is the shovel pointing to your left or to your right?"; Pedestrian Crossing, "At a pedestrian-controlled traffic lights, there is a display for pedestrians operated by a push-button. The signal to cross is the illumination of a small green figure. Is the person shown as walking to your left or to your right?"

After the memory probes, which were part of a larger session, handedness was elicited as follows: "Would you describe yourself as left-handed or right-handed? In particular, which hand do you use for drawing?" Participants then completed the handedness questionnaire of M. Annett (1985).

\section{Results}

For the small number of participants with incomplete memory responses, those responses that they did make were included in the analyses reported here; participants with incomplete handedness questionnaire responses were excluded from relevant analyses.

Road Works. The proportion of correct recall for the Road Works sign (i.e., left responses) for right-handed participants $(64.0 \%)$ was significantly higher than that for left-handed participants $(44.2 \%)\left[\chi^{2}(1, N=514)=\right.$ $19.52, p<.001]$. The level of correct recall was significantly above the guessing level of $50 \%$ for right-handed participants $\left[\chi^{2}(1, N=317)=24.99, p<.001\right]$ but did not differ significantly from it for left-handed participants $\left[\chi^{2}(1, N=197)=2.69\right]$.

The advantage of right-handed over left-handed participants was examined further in order to determine whether it held both for participants exhibiting pure handedness and for those exhibiting mixed handedness. All participants who completed the M. Annett (1985) questionnaire and did not respond either for writing-hand preference were included. The proportion of correct left responses for pure right-handers was significantly greater than that for pure left-handers $\left[\chi^{2}(1, N=326)=19.77, p<\right.$ $.001]$. For pure right-handers, the proportion of correct responses was $64.0 \%$, which was significantly above the chance level of $50 \%\left[\chi^{2}(1, N=236)=18.46, p<.001\right]$. The same proportion for pure left-handers was $36.7 \%$, which was significantly below the $50 \%$ level $\left[\chi^{2}(1, N=\right.$ $90)=6.40, p<.05]$. For participants of mixed handedness, the difference between right-handers and left-handers was in the same direction but of smaller magnitude; response levels for both right-handers and left-handers were less extreme for groups of mixed handedness than for those of pure handedness. For mixed right-handers and mixed left-handers, the proportions of correct responses were $62.8 \%$ and $50.0 \%$, respectively, and did not differ significantly $\left[\chi^{2}(1, N=180)=2.94\right]$.

The relation between degree of handedness and recall was also examined by regression analysis. Recall scores were 0 and 1 for right and left responses, respectively, and the handedness index ranged from 12 (complete right) to 36 (complete left). There was a significant linear regression of recall score on the handedness index $[F(1,509)=$ $20.03, p<.001]$ but no significant quadratic or higher trend. If $\rho$ and $\alpha$ represent proportion of correct recall and handedness, respectively, the best-fitting equation was $\rho=-.0104 \alpha+.779$. For comparing performance across conditions and across experiments, it is also convenient to adopt an alternative, uniform response code of 0 and 1 for left and right responses, respectively, irrespective of whether the left or the right response is the correct one. If the coding of the left and right responses is reversed in this way, the gradient of the new regression equation is the reverse of the old one, and its intercept is the complement of the old one (i.e., 1 minus the old intercept). Thus, if $\theta$ represents the resulting proportion of right responses, the regression equation in this case is $\theta=.0104 \alpha+.221$.

Pedestrian Crossing. The proportion of correct recall for the Pedestrian Crossing signal (i.e., right responses) for left-handed participants $(59.6 \%)$ was significantly higher than that for right-handed participants $(48.2 \%)$ $\left[\chi^{2}(1, N=511)=6.27, p<.05\right]$. The level of correct recall was significantly above the guessing level of $50 \%$ for left-handed participants $\left[\chi^{2}(1, N=198)=7.29, p<.01\right]$ but did not differ significantly from it for right-handed participants $\left[\chi^{2}(1, N=313)=0.39\right]$.

The advantage of left-handed over right-handed participants was examined further to determine whether it held both for participants exhibiting pure handedness and for those exhibiting mixed handedness. The proportion of correct right responses for pure left-handers was significantly greater than that for pure right-handers $\left[\chi^{2}(1, N=324)=8.56, p<.01\right]$. For pure left-handers, the proportion of correct responses was $65.6 \%$, which was significantly above the chance level of $50 \%\left[\chi^{2}(1\right.$, $N=90)=8.71, p<.01]$. The same proportion for pure right-handers was $47.4 \%$, which did not differ significantly from the $50 \%$ level $\left[\chi^{2}(1, N=234)=0.62\right]$. For participants of mixed handedness, the difference between left-handers and right-handers was in the same direction but of smaller magnitude; response levels for both left-handers and right-handers were less extreme for groups of mixed handedness than for those of pure handedness. For mixed left-handers and mixed right-handers, the proportions of correct responses were $53.4 \%$ and $49.4 \%$, respectively, and did not differ significantly $\left[\chi^{2}(1, N=180)=0.29\right]$.

The relation between degree of handedness and recall was also examined by regression analysis. In this condition, recall scores were 0 and 1 for left and right responses, respectively, and the handedness index ranged, as before, from 12 (complete right) to 36 (complete left). There was a significant linear regression of recall score on handedness index $[F(1,507)=9.53, p<.01]$ but no significant quadratic or higher trend. If $\rho$ and $\alpha$ represent proportion of correct recall and handedness, respectively, the bestfitting equation was $\rho=.00730 \alpha+.376$. In this condition, the regression equation for $\theta$, the proportion of right responses, is of course identical to that for $\rho$. 


\section{Discussion}

The results of this experiment provide decisive evidence in favor of the compatibility hypothesis and against the group ability hypothesis. In the Pedestrian Crossing condition, with a right-facing stimulus, left-handed people performed significantly better than right-handed people. This is the same pattern as that found previously for the recall of coin design. In the Road Works condition, however, with a left-facing stimulus, the position was reversed, and right-handed people performed significantly better than left-handed people. That is, neither left-handed nor right-handed people exhibit a general superiority in memory for orientation, but instead their relative levels of performance depend on the particular characteristics of the task being performed.

To explore further the compatibility hypothesis, is it possible to account in detail for the observed pattern of superior recall for a right-facing stimulus by left-handed people and superior recall for a left-facing stimulus by right-handed people? It is proposed here that motor imagery underlies this observed pattern. Motor imagery refers to the phenomenon that some of the psychological processes that are activated by movement may also become activated in the absence of actual movement (see, e.g., J. Annett, 1995; Jeannerod, 1994, 1997). It is suggested that, even when recall is verbal, as in the present experiment, motor processes are mobilized that may influence the product of recall. There is evidence (Shanon, 1979) that left-handed people have a tendency to draw heads facing to the right and vice versa. This may be because people tend to draw fine details initially and then move the hand in the direction that allows them to draw the remainder without obscuring the initial work. Thus, right-handed people may tend to draw a left-facing profile because they first draw the facial details and then move the hand away to the right to draw the back of the head. It is possible, therefore, that the effects of handedness in the present experiment reflect similarly lateralized effects that occur when drawing figures, mediated through motor imagery. A further experiment was thus carried out to investigate whether the drawing of figures in fact exhibits effects of handedness similar to those observed in recall.

\section{EXPERIMENT 3}

\footnotetext{
Method

Participants. There were 230 new participants. Categorized on the basis of the hand used for drawing, 111 were left-handed, and 119 were right-handed. They were volunteers who were students or interviewees at $O x$ ford, with average age 19.4 years $(S D=1.7$ years).

Procedure. Each participant was tested using a question booklet with successive items printed on separate pages. As part of a larger session, half of the participants drew a person digging first and a person walking second; for the other half, the order was reversed. The participants were instructed to draw inside frames that were $4 \mathrm{~cm}$ square, as follows: "Please draw a quick sketch of a man digging a hole with a spade [or, a man walking]. Please draw inside the square provided."
}

After the drawing, handedness was elicited as follows: "Would you describe yourself as left-handed or right-handed? In particular, which hand do you use for drawing?" The participants then completed M. Annett's (1985) handedness questionnaire.

\section{Results}

The small number of incomplete responses were treated in a manner similar to that in Experiment 2.

The data were analyzed separately for the digging and walking conditions. For both conditions, the proportion of right-facing drawings was significantly higher for lefthanded participants (digging $76.4 \%$, walking $78.7 \%$ ) than for right-handed participants (digging $53.5 \%$, walking $62.1 \%$ ) [digging $\chi^{2}(1, N=224)=12.81, p<.001$; walking $\left.\chi^{2}(1, N=224)=7.38, p<.01\right]$. For both conditions, the level of right-facing drawing for left-handed participants was significantly above the chance level of $50 \%$ [digging $\chi^{2}(1, N=110)=30.58, p<.001$; walking $\left.\chi^{2}(1, N=108)=35.59, p<.001\right]$. The level of rightfacing drawing for right-handed participants did not differ from the chance level for digging $\left[\chi^{2}(1, N=114)=\right.$ $0.56]$ but was significantly above it for walking $\left[\chi^{2}(1\right.$, $N=116)=6.76, p<.01]$.

The difference in performance between left-handed and right-handed participants was examined further to determine whether it held both for participants exhibiting pure handedness and for those exhibiting mixed handedness. For both conditions, the proportion of right-facing responses for pure left-handers was significantly greater than that for pure right-handers [digging $\chi^{2}(1, N=$ $110)=4.71, p<.05$; walking $\chi^{2}(1, N=110)=5.89$, $p<.05]$. For both conditions, the proportion of right-facing responses for pure left-handers (digging $66.7 \%$, walking $79.5 \%$ ) was significantly above the chance level of $50 \%$ [digging $\chi^{2}(1, N=39)=4.33, p<.05$; walking $\left.\chi^{2}(1, N=39)=13.56, p<.001\right]$. For both conditions, the same proportion for pure right-handers (digging $45.1 \%$, walking $56.3 \%$ ) did not differ significantly from the $50 \%$ level [digging $\chi^{2}(1, N=71)=0.69$; walking $\left.\chi^{2}(1, N=71)=1.14\right]$.

For both conditions, the difference in level of rightfacing response between left-handers and right-handers was in the same direction for participants of mixed handedness as for participants of pure handedness but of smaller magnitude; for walking, but not digging, response levels for both left-handers and right-handers were less extreme for groups of mixed handedness than for those of pure handedness. For both conditions, the proportions of right-facing responses for mixed left-handers (digging $81.7 \%$, walking $77.3 \%$ ) and mixed right-handers (digging $68.3 \%$, walking $72.1 \%$ ) did not differ significantly [digging $\chi^{2}(1, N=112)=2.61$; walking $\chi^{2}(1, N=$ 109) $=0.55$ ]. Indeed, for both conditions, pure left-handers, mixed left-handers, and mixed right-handers did not differ significantly from each other in their proportions of right-facing responses [digging $\chi^{2}(2, N=$ $151)=3.98$; walking $\left.\chi^{2}(2, N=148)=0.68\right]$ but col- 
lectively differed from pure right-handers [digging $\chi^{2}(1$, $N=222)=17.95, p<.001$; walking $\chi^{2}(1, N=219)=$ $9.13, p<.01]$.

The relation between degree of handedness and drawing direction was also examined by regression analysis. Scores were 0 and 1 for left-facing and right-facing responses, respectively, and the handedness index ranged, as before, from 12 (complete right) to 36 (complete left). For both conditions, there was a significant linear regression of response score on handedness index [digging $F(1,221)=10.01, p<.01$; walking $F(1,221)=5.42, p<$ $.05]$ but no significant quadratic or higher trend. If $\theta$ and $\alpha$ represent proportion of right-facing responses and handedness, respectively, the best-fitting equations were $\theta=.0109 \alpha+.408$ and $\theta=.00778 \alpha+.532$ for digging and walking, respectively.

\section{Discussion}

It was found that the way in which handedness influenced the drawing of a figure was closely similar to the way in which it influenced the verbal recall of the figure. This finding provides direct support for the motor imagery hypothesis of handedness effects in recall. Further implications are discussed in the following section.

\section{GENERAL DISCUSSION}

The results of all three experiments reported here demonstrate that, contrary to the fears expressed by $\mathrm{Ba}$ naji and Crowder (1989), findings observed in one domain of everyday memory can generalize fully to another domain. Two striking characteristics of memory for the appearances of coins (Jones, 1990; Jones \& Martin, 1997; McKelvie \& Aikins, 1993; Nickerson \& Adams, 1979) are the low overall level of recall despite repeated exposure over many years and the dependency of one type of recall on the handedness of the person recalling. Both patterns of response were observed in the present experiments to characterize memory for road signs also.

Experiment 1 showed that the first of the coin findings (namely, the failure to remember that which has been viewed very often) is as pronounced for road signs as it is for coins. Jones (1990) identified four types of problems that cause low levels of recall-namely, low levels of meaningfulness, redundancy, identifiability, and discriminativeness. As an example, the octagon shape of the Stop sign possesses little meaningfulness (i.e., octagons have relatively few semantic predicates) and no redundancy (i.e., there is no schema relating road signs to octagons, since the Stop sign is the only octagonal sign). Thus, even though the octagon can be readily identified and serves to discriminate well among signs, it was correctly recalled by only a small proportion of participants $(20 \%)$. Considerably more participants $(49 \%)$ incorrectly recalled the Stop sign as circular, inappropriately utilizing the schema that many British road signs that give orders (e.g., No Entry) are circular in shape.
Turning to handedness, the present results with road signs confirm and substantially extend previous findings with coins. The observation in Experiment 2 of recall advantages for both left-handed and right-handed participants, depending on what is being recalled, provides decisive evidence in favor of an explanation of handedness effects on memory in terms of compatibility between group and task rather than in terms of group ability per se. It may also be possible to feed back to the area of memory for coins the finding of left-handed superiority for recall of a right-facing figure and right-handed superiority for a left-facing figure. In particular, it is possible that an interaction between denomination and handedness will be found to occur in the recall of coinages (e.g., that of the United States) in which a head can face either to the right (the U.S. penny) or to the left (other U.S. coins).

The close correspondence between the pattern of handedness dependence observed in verbal recall (Experiment 2) and in drawing (Experiment 3 ) provides support for the hypothesis that handedness effects observed in verbal recall are the consequence of underlying motor imagery processes. The results of these two experiments provide evidence also that handedness effects in recall are not all-or-none but are instead graded, with variation in the magnitude of the effects across individuals reflecting graded measures of handedness rather than simply dichotomous ones. This may be illustrated by the regression information shown in Table 2.

The regression gradients shown in Table 2 summarize in quantitative form the manner in which a pseudocontinuous measure of handedness (viz., total numerical score on the $M$. Annett questionnaire) determines whether a person produces a left or a right response in two different tasks (viz., verbal recall in Experiment 2 and drawing in Experiment 3). It can be seen that, for both types of stimuli studied (relating to digging and to walking), there was a close match between the gradients observed in the two experiments. The striking similarity between the results for recall and for drawing suggests that the performance of either task activates common underlying processes that are sensitive to handedness. Alternatively expressed, handedness-dependent motor imagery in the recall task appears to echo handedness-dependent motor articulation in the drawing task.

Further evidence on the issue of a graded as opposed to a dichotomous approach to handedness was provided when the participants were divided in these experiments

Table 2

Gradient of Linear Regression of Proportion of Right Responses on Handedness Score, for Recalled and for Drawn Responses

\begin{tabular}{lcccc}
\hline \multicolumn{2}{c}{ Response } & & & \multicolumn{2}{c}{ Gradient $\left(\times 10^{-3}\right)$} \\
\cline { 5 - 6 } Recalled & Drawn & & Recalled & Drawn \\
\hline Road Works & Digging & & 10.4 & 10.9 \\
Pedestrian Crossing & Walking & 7.3 & 7.8 \\
\hline
\end{tabular}


into pure and mixed handedness groups. Following M. Annett $(1985,1992)$, overall handedness corresponded to the hand used for writing; the pure group used that hand either predominantly or at least equally for all the other activities sampled, whereas the mixed group used the other hand predominantly for at least one of the other activities. It was found that the two pure groups differed significantly from each other in terms both of what they recalled and of what they drew, in all conditions. In contrast, the two mixed groups did not differ significantly from each other in any condition. Furthermore, response levels were numerically less extreme for groups of mixed handedness than for groups of pure handedness in all except one condition (namely, drawing a man digging, for which there was no significant difference between groups of mixed handedness and of pure left-handedness). Overall, these patterns tend to imply that there was a genuine functional significance attached to the distinction between participants with pure handedness and those with mixed handedness and thus provide support for a graded view of handedness.

It should be noted that the motor imagery processes hypothesized to be instrumental in mediating handedness effects in recall need not be available to conscious introspection. Existing evidence indicates that at least some aspects of introspection concerning imagery do not provide a reliable guide to behavior. For example, the introspective vividness of motor imagery does not appear to influence observed behavior in a consistent way (see J. Annett, 1995). Following Jeannerod (1994, 1997), motor imagery is defined here, not in terms of the subjective experience of movement in the absence of real movement, but instead in terms of the activation of movement processes in the absence of overt movement. It is proposed that, even when the output of recall is entirely verbal, motor imagery may, in some cases, be activated during the retrieval stage. The task of deciding whether a person faced to the left or the right is one such case. The motor imagery thus activated shares processing elements with those underlying overt drawing behavior. Hence, differences in overt drawing between left-handed and righthanded people (as reported both here and by Shanon, 1979) can be reflected also in verbal recall. The hypothesized motor imagery may, in principle, be investigated further in a number of ways - either by means of behavioral techniques such as dual-task interference (see, e.g., Logie, 1995) and developmental fractionation (see, e.g., Logie \& Pearson, 1997) or by direct cortical activity mapping (see, e.g., Cohen et al., 1996).

In conclusion, the results of the present experiments have demonstrated the generalizability of everyday memory. Memory for road signs closely resembles memory for coins. But these results have also extended substantially our understanding of handedness effects in verbal recall. They show that the effects of handedness reflect the compatibility between group and task rather than simple differences between groups, and they implicate motor imagery as the underlying mediator of these effects.

\section{REFERENCES}

AnNetT, J. (1995). Motor imagery: Perception and action. Neuropsychologia, 33, 1395-1417.

ANNETT, M. (1985). Left, right, hand and brain: The right shift theory. London: Erlbaum

ANNETT, M. (1992). Spatial ability in subgroups of left- and righthanders. British Journal of Psychology, 83, 493-515.

BANAJI, M. R., \& Crowder, R. (1989). The bankruptcy of everyday memory. American Psychologist, 44, $1185-1193$.

Bishop, D. V. M. (1990). Handedness and developmental disorder. Oxford: Blackwell.

BOWER, G. H. (1994). A turning-point in mathematical learning-theory. Psychological Review, 101, 290-300.

Bryden, M. P., MCManus, I. C., \& Bulman-Fleming, M. B. (1994). Evaluating the empirical support for the Geschwind-BehanGalaburda model of cerebral lateralization. Brain \& Cognition, 26, 103-167.

BRYSON, B. (1991). Mother tongue: The English language. London: Penguin. [Originally published (1990) as The mother tongue: English \& how it got that way. New York: William Morrow.]

Cohen, M. S., Kosslyn, S. M., Breiter, H. C., Digirolamo, G. J., Thompson, W. L., ANDERSON, A. K., BoOKHeIMER, S. Y., RoSEN, B. R., \& Belliveau, J. W. (1996). Changes in cortical activity during mental rotation: A mapping study using functional MRI. Brain, 119, 89-100.

DEPARTMENT OF TRANSPORT (1989). Know your traffic signs (3rd ed.). London: HMSO.

Eglinton, E., \& Annett, M. (1994). Handedness and dyslexia: A meta-analysis. Perceptual \& Motor Skills, 79, 1611-1616.

Geschwind, N., \& BEHAN, P. (1982). Left-handedness: Associations with immune disease, migraine, and developmental learning disorder. Proceedings of the National Academy of Sciences, 79, 5097-5100.

JEANNEROD, M. (1994). The representing brain: Neural correlates of motor intention and imagery. Behavioral \& Brain Sciences, 17, 187-245.

JEANNEROD, M. (1997). The cognitive neuroscience of action. Oxford: Blackwell.

JoNES, G. V. (1976). A fragmentation hypothesis of memory: Cued recall of pictures and of sequential position. Journal of Experimental Psychology: General, 105, 277-293.

JoNES, G. V. (1990). Misremembering a common object: When left is not right. Memory \& Cognition, 18, 174-182.

Jones, G. V., \& MARTiN, M. (1997). Handedness dependency in recall from everyday memory. British Journal of Psychology, 88, 609-619.

LEVY, J. (1969). Possible basis for the evolution of lateral specialization of the human brain. Nature, 224, 614-615.

LoGIE, R. H. (1995). Visuo-spatial working memory. Hillsdale, NJ: Erlbaum.

Logie, R. H., \& Pearson, D. G. (1997). The inner eye and the inner scribe of visuo-spatial working memory: Evidence from developmental fractionation. European Journal of Cognitive Psychology, 9, 241-257.

LuH, K. E., REDL, J., \& LEVY, J. (1994). Left- and right-handers see people differently: Free-vision perceptual asymmetries for chimeric stimuli. Brain \& Cognition, 25, 141-160.

Martin, M., \& Jones, G. V. (1995). Danegeld remembered: Taxing further the coin head illusion. Memory, 3, 97-104.

McKelvie, S. J., \& AIKINS, S. (1993). Why is coin head orientation misremembered? Tests of schema interference and handedness hypotheses. British Journal of Psychology, 84, 355-363.

Mebert, C., \& MiCHEL, G. (1980). Handedness in artists. In J. Herron (Ed.), Neuropsychology of left handedness (pp. 273-279). New York: Academic Press.

NiCKERSON, R. S., \& ADAMS, M. J. (1979). Long-term memory for a common object. Cognitive Psychology, 11, 287-307.

Rubin, D. C., \& KonTIS, T. C. (1983). A schema for common cents. Memory \& Cognition, 11, 335-341.

SHANON, B. (1979). Graphological patterns as a function of handedness and culture. Neuropsychologia, 17, 457-465.

SHEPARD, R. N. (1967). Recognition memory for words, sentences, and pictures. Journal of Verbal Learning \& Verbal Behavior, 6, 156-163. 
SPRINGER, S. P., \& DeUTSCH, G. (1993). Left brain, right brain (4th ed.). New York: Freeman.

Standing, L. (1973). Learning 10,000 pictures. Quarterly Journal of Experimental Psychology, 25, 207-222.

\section{NOTE}

1. "In a rather more roundabout way, so to speak, the word roundabout, the British term for traffic circles, is of American origin. More precisely, it was a term invented by Logan Pearsall Smith, an American living in England, who was one of the members in the 1920s of the BBC Advisory Committee on Spoken English. This lofty panel had the job of deciding questions of pronunciation, usage, and even vocabulary for the BBC. Before Smith came along, traffic circles in Britain were called gyratory circuses." (Bryson, 1991, p. 165)

(Manuscript received July 21, 1997; accepted for publication October 8, 1997.) 